\title{
Analysis on adverse reactions caused annually by cases of antibiotic use in a hospital of China
}

\author{
Wei Niu', Lian-yi Guo ${ }^{1 \star}$, Xu-peng Jin², Gui-jun Wang ${ }^{1}$, Xuan Shi ${ }^{1}$ and Bao-hai Liu ${ }^{1}$ \\ ${ }^{1}$ Department of Gastroenterology, the First Affiliated Hospital of Liaoning Medical College, Jinzhou 121001, Liaoning \\ Province, China. \\ Liaoning Medical College, Jinzhou 121001, Liaoning Province, China.
}

Accepted 13 June, 2012

\begin{abstract}
This study aims to investigate adverse reactions caused by antibiotics, and promote clinically safe, effective and rational use of antibiotics. Data on antibiotic use were obtained from the First Affiliated Hospital of Liaoning Medical College of China from September 2009 to May 2010, summarized and statistical analyses were carried out respectively on antibiotic dosage, times of adverse reactions caused by antibiotics, drug administration routes and drug forms. The findings of this research revealed that antibiotics have many adverse reactions, but they can be prevented and controlled, and we should pay attention to clinical monitoring during drug use. Hence only safe, effective and rational use of antibiotics can effectively show the efficacy and reduce the incidence of adverse reactions.
\end{abstract}

Key words: Antibiotics, adverse reaction, clinical application.

\section{INTRODUCTION}

In clinical practice, antibiotics are the most commonly used drugs in recent years, accompanied by the increase of antibiotic type and dosage forms, the occurrence of adverse drug reactions frequently reported, so drug safety is an important problem in the clinical application process. Data on antibiotic use were obtained from the First Affiliated Hospital of Liaoning Medical College of China from September 2009 to May 2010.

\section{METHODOLOGY}

Records of 6900 cases of antibiotic use and that of adverse reactions were collected from the First Affiliated Hospital in September 2009 to May 2010. To summarize the data of antibiotic use in the Department of Pharmacy in our hospital in September 2009 May 2010, and make statistical analysis respectively on antibiotic dosage, times of adverse reactions caused by antibiotics, drug administration routes and drug forms according to Clinical use of antibiotics management policies (Chinese Ministry of Health).

\footnotetext{
*Corresponding author. E-mail: angel_gly@yahoo.com.cn.
}

\section{RESULTS}

Drug varieties and use frequency

Amoxicillin in penicillins, cefuroxime in second-generation cephalosporins, azithromycin in macrolides and compound preparations of Augmentin and Sulbactam in $\beta$-lactamase inhibitors exceed 500 times of use, in which, compound preparations are up to 575 times. $\beta$-lactams take a dominant position in clinical drug use today and the next is macrolides, aminoglycosides, such as gentamicin, are often used in the Department of Gastroenterology, and tetracyclines, chloramphenicols and others have been mostly phased out due to serious side effects and for external use, as shown in Table 1.

Clinical manifestations of adverse reactions caused by antibiotics

The adverse reactions caused by antibiotics involved many organs and systems, mainly including allergic 
Table 1. Drug varieties and use frequency.

\begin{tabular}{llc}
\hline Variety & Drug example & Dosage unit / time \\
\hline & Penicillin VK (Penicillins) & 112 \\
& Amoxicillin (Penicillins) & 518 \\
& Ampicillin (Penicillins) & 450 \\
& Cefradine (First-generation Cephalosporins) & 387 \\
B-lactams & Cefalexin (First-generation Cephalosporins) & 463 \\
& Cefuroxime (Second-generation Cephalosporins) & 538 \\
& Cefaclor (Second-generation Cephalosporins) & 422 \\
& Cefoperazone (Third-generation Cephalosporins) & 441 \\
& Cefixime (Third-generation Cephalosporins) & 119 \\
& Cefpirome (Fourth-generation Cephalosporins) & 55 \\
& Augmentin (Compound preparation) & 530 \\
& Sulbactam (Compound preparation) & 575 \\
Macrolides & & \\
& Roxithromycin (14-membered ring) & 111 \\
Aminoglycosides & Azithromycin (15-membered ring) & 570 \\
Tetracyclines & Acetylspiramycin (16-membered ring) & 459 \\
Chloramphenicols & Gentamicin & 95 \\
Others & Terramycin & 34 \\
\hline
\end{tabular}

reactions, neurotoxicity, hepatotoxicity, renal toxicity and superinfection, as shown in Table 2 . The allergic reaction was mainly caused by penicillin and cephalosporin. Impurity in the penicillin and its metabolites is a semiantigenic material (thiazolyl acyl form), which bind tissue protein to form antigen in the blood, and formation of the whole antigen can induce the body to produce $\lg \mathrm{E}$ antibodies.

\section{Drug administration routes}

The common drug administration routes are oral administration, subcutaneous injection and intravenous infusion, as shown in Table 3.

We found that adverse drug reactions caused by intravenous infusion up to $63.52 \%$, intravenous infusion was the main formulations of the antibiotic drugs causing adverse reactions.

\section{Drug forms}

The common drug forms are tablet, capsule, injection, etc, as shown in Table 4. We found that different forms of a particular drug cause different absorption bioavailability, and improper drug dosage can also cause adverse reactions.

\section{DISCUSSION}

\section{Relationship between drug varieties and adverse reactions}

It can be seen from Table 1 that the commonly used antibiotics in clinical are primarily $\beta$-lactams and secondarily macrolides and aminoglycosides. We should pay attention to the compatibility of drugs in the use of antibiotics, to avoid the synergistic effects of virulence (Hai-ying et al., 2006). The combination of cephalosporins with other nephrotoxic drugs may aggravate kidney damage, such as aminoglycosides and potent diuretics; two aminoglycoside antibiotics should not be used at same time or continuously locally or systemically used, otherwise it will increase ototoxicity (Xin-min Liu, 2010).

\section{Relationship between drug administration routes and adverse reactions}

For drug administration routes, intravenous infusion is the main route causing adverse drug reactions. Doctors usually adopt intravenous infusion for inpatients, as shown in Tables 2 and 3. In this report, 3811 cases of adverse drug reactions are caused by intravenous infusion, accounting for $63.52 \%$ of the total, followed by 
Table 2. Clinical manifestations of adverse reactions caused by antibiotics and number of cases.

\begin{tabular}{|c|c|c|c|c|c|c|c|}
\hline Antibiotics & Penicillins & Cephalosporins & Aminoglycosides & Macrolides & Tetracyclines & Chloramphenicols & Others \\
\hline Allergic shock & 2 & 1 & 1 & 0 & 0 & 0 & 0 \\
\hline Serum disease & 7 & 5 & 1 & 0 & 0 & 0 & 0 \\
\hline Drug fever & 9 & 6 & 0 & 0 & 0 & 1 & 1 \\
\hline Allergic reaction & 96 & 221 & 5 & 38 & 2 & 0 & 0 \\
\hline Neurotoxicity & 56 & 106 & 46 & 76 & 10 & 5 & 2 \\
\hline Renal toxicity & 0 & 59 & 66 & 0 & 0 & 0 & 3 \\
\hline Hepatotoxicity & 4 & 26 & 0 & 69 & 9 & 3 & 2 \\
\hline Blood toxicity & 3 & 39 & 0 & 0 & 0 & 40 & 1 \\
\hline Gastrointestinal toxicity & 40 & 95 & 0 & 621 & 12 & 10 & 3 \\
\hline Immunotoxicity & 0 & 26 & 0 & 0 & 11 & 4 & 2 \\
\hline Cardiotoxicity & 26 & 0 & 0 & 0 & 0 & 0 & 1 \\
\hline Specific reaction & 0 & 0 & 0 & 0 & 0 & 2 & 1 \\
\hline Superinfection & 10 & 37 & 0 & 0 & 12 & 5 & 0 \\
\hline
\end{tabular}

Table 3. Drug administration routes and proportions.

\begin{tabular}{lcc}
\hline Drug administration route & $\mathbf{n}$ & Proportion (\%) \\
\hline Intravenous infusion & 3811 & 63.52 \\
Oral administration & 1372 & 22.87 \\
Intramuscular injection & 315 & 5.25 \\
Intravenous injection & 301 & 5.02 \\
Subcutaneous injection & 102 & 1.70 \\
Local injection & 32 & 0.53 \\
Intraperitoneal drug use & 14 & 0.23 \\
Intrauterine drug use & 18 & 0.30 \\
Local external use & 21 & 0.35 \\
Intracutaneous injection & 14 & 0.23 \\
Total & 6000 & 100.00 \\
\hline
\end{tabular}

1372 cases of oral administration, accounting for $22.87 \%$ of the total. Intravenously infused drugs directly enter blood vessels and take effect rapidly, while oral drugs are digested, absorbed and screened in intestinal tract, which also increases the incidence of adverse drug reactions caused by intravenous infusion (Su-jiao and Liang, 2008).

The difference in drug administration routes is related to drug absorption and distribution and also affects the speed, intensity and duration of drug efficacy, intravenous drugs directly enter blood circulation and take effect immediately, 
Table 4. Analysis on adverse antibiotic reactions and drug forms.

\begin{tabular}{lcccccccc}
\hline Analysis & $\begin{array}{c}\text { Powder } \\
\text { injection }\end{array}$ & Injecta & Tablet & Capsule & Powder & $\begin{array}{c}\text { Controlled } \\
\text { release form }\end{array}$ & Spray & Others \\
\hline Total times of use & 3876 & 12109 & 18953 & 790 & 77 & 9052 & 421 & 7 \\
Times of adverse reactions & 143 & 451 & 13 & 19 & 2 & 19 & 7 & 0 \\
Percentage & 3.68 & 3.70 & 0.68 & 2.40 & 2.59 & 0.20 & 1.66 & 0 \\
\hline
\end{tabular}

prone to adverse reactions, and oral stimulant drugs can cause nausea, vomiting, etc. In principle, oral administration is better than intravenous infusion in treatment, and safe and effective treatment should be provided (Ren-wei Shi, 2011).

\section{Relationship between drug forms and adverse reactions}

Different forms of a particular drug cause different absorption in body, that is, difference bioavailability, and improper drug dosage can also cause adverse reactions. It can be seen from Table 4 that intravenous infusion has maximum bioavailability can causes most adverse reactions, followed by powder injection, and tablets are used frequently, but its bioavailability is limited, so the incidence of adverse reactions is significantly lower (Zheng-chun et al., 2004).

\section{Preventive measures for adverse antibiotic reactions}

Medical care staff should be familiar with the "Clinical antibiotic application guide and principles" for drug use instructions (Bin et al., 2001; Amsden, 2005), master the indications, contraindications, pharmacokinetics and adverse reactions of antibiotics (Wen-zhen, 2004), rationally use drugs according to the physiological state of patients. For example, pregnant women should cautiously use or not use the drugs that is able to pass through placental barrier (Jian-cheng Liang, 2005); patients with liver or renal insufficiency and elderly patients should use drug with caution (Lanzoni et al., 2005). If narrow-spectrum antibiotics are effective, do not use broad-spectrum antibiotics (Li-xin and Xiu-mei, 2004). Select appropriate dosage regimens, drug administration routes, dosage and treatment course, and if necessary, combine with plasma concentration for monitoring (Ding and Salvi, 2005; Hua-cheng and Li-shi, 2005). Patients should actively cooperate with the treatment and tell doctors about family history, allergic history and other situations, so that doctors can make quick and accurate diagnosis (Zhong-wei, 2005). Clinical pharmacists should pay attention to ADR reporting and monitoring work to promote clinical rational use of antibiotic drugs, and find more drug safety information in order to explore ADR mechanism in depth (Zhi-qiang, 2006).

In conclusion, $\beta$-lactam and macrolide antibiotics are the most widely used in clinical, but antibiotics cause allergic reactions, toxic reactions and specific reactions, superinfection and other adverse reactions, and drug administration routes and drug forms can also cause the occurrence of adverse reactions. Therefore, we should rationally and specifically use antibiotics and minimize the adverse reactions in treatment. Doctors should be familiar with the types, contraindications and adverse reactions of the antibiotics used, clarify the medication purposes before drug use, and learn about patient's conditions and severity, so as to specifically select drug and its form and dosage and make detailed, rational and effective regimens. Once adverse reactions are found, take immediate rescue measures. Only in this way, we can achieve the purpose of effective and rapid treatment.

\section{REFERENCES}

Amsden GM (2005). Anti.inflammatory effects of macrolides.an underappreciated benefit in the treatment of community.acquired respiratory tract infections and chronic inflammatory pulmonary conditions. Antimicrob. Chemother. 55(1):10.

Bin L, Hong-jun Peng (2001). Analysis on 136 cases of drug-induced hepatitis. Chinese J. Pharmacoepidemiol. 3 (5):251-253.

Ding D, Salvi R (2005). Review of cellular changes in the cochlea due to aminoglycoside antibiotics. The Volta Rev. 105(3):407-438.

Hai-ying Z, Sheng-dao X, Weining X (2006). Comparative study on antibiotic monotherapy or combination therapy inducing pseudomonas aeruginosa to release endotoxin. Chinese J. Microbiol. Immunol. 26(1):18-22.

Hua-cheng $\mathrm{H}$, Li-shi $\mathrm{L}$ (2005). Adverse reactions caused by aminoglycoside antibiotics. Chinese J. Postgraduates Med. 28 (11): 3-4.

Jian-cheng L, Yi-kun H (2005), Zhang Sanping. Analysis on 161 cases of adverse drug reactions. Anthol. Med. 24 (2):48-49.

Lanzoni I, Corbacella E, Ding D (2005). MDL 28170 attenuates gentamicin ototoxicity. Audiol. Med. 3(2): 82-89.

Li-xin Z, Xiu-mei W (2004). Problems in antibiotic application and exploration. J. Practical Med. Techniques 11 (8):1498-1499.

Ren-wei S (2011). Analysis on risks in clinical drug use. Evaluation and Analysis of Drug-use in Hospitals of China 11(1):92-93.

Su-jiao T, Liang $Z$ (2008). Analysis on intravenous antibiotic use in outpatient department in primary hospitals. Chinese J. Drug Applicat. Monitoring 5(5):39-40.

Wen-zhen W, Jian-hui L (2004). Clinical analysis on 220 cases of drug rash. Modern J. Integrated Traditional Chinese Western Med. 13 (13): 1739

Xin-min L (2010). Analysis on clinical irrational drug combination of aminoglycoside antibiotics [J]. Chinese J. Modern Drug Applicat. 4 (24): 150-151.

Zheng-chun W, LiQ, Wang S (2004). Analysis on 803 cases of 
adverse drug reactions . Herald Med. 23 (9):695-696.

Zhi-qiang $L$ (2006). Adverse reactions and rational use of antibiotics. J. Clinical Experimental Med. 5(7):1030.
Zhong-wei T (2005). Antibiotic therapy for bacterial infection in respiratory tract. Chinese J. Practical Internal Med. 25 (2):113. 